\title{
OPTICAL TOMOGRAPHY OF THE IN VIVO HUMAN LENS: THREE-DIMENSIONAL VisUALIZATION OF CATARACTS
}

\author{
Barry R. Masters \\ Uniformed Services University of the Health Sciences, Department of Anatomy and Cell Biology, \\ 4301 Jones Bridge Road, Bethesda, Maryland 20814-4799 \\ (Paper JBO/LSF-07 received March 25, 1996; revised manuscript received May 14, 1996; accepted for publication May 15, \\ 1996)
}

\begin{abstract}
An in vivo human lens containing a cataract has been visualized by a series of orthogonal slices made through a three-dimensional volume reconstruction. Data acquisition was made with a transformed series of 60 rotated Scheimpflug digital images. Each digital image represents the light scatter from the lens in a plane that contains the optic axis. At each angular position of the camera, a digital image of the in vivo ocular lens was acquired. The set of 60 Scheimpflug digital images was mathematically transformed into a new data set in which the images were oriented perpendicular to the optic axis of the eye. The transformed set of optical sections was aligned to correct for small eye movements during the data collection process. In order to visualize the volume of the in vivo human lens, slices were projected through the lens volumetric data set. The use of orthogonal slices to visualize lenticular light scatter represents a new technique to visualize human cataracts in vivo in three dimensions. () 1996 Society of Photo-Optical Instrumentation Engineers.
\end{abstract}

Keywords light scattering and fluorescence; optical tomography; in vivo human lens; light scatter, cataracts.

\section{INTRODUCTION}

Light scatter is an integral part of our lives. From the blue sky to the beautiful red sunset, we see the effects of light scatter. The names of Tyndall, Lord Rayleigh, Debye, Mie, and Einstein are associated with contributions in this field. Light scattering techniques are used to study the size, shape, and molecular dynamics of scattering particles. Increases in the light scatter from the human lens are a normal part of the aging process, and techniques have been developed to use the light scattering from the lens to investigate the location, size, and structure of these scattering centers.

The human lens is a refractive element of the eye with the ability to vary its power (accommodation). The lens is a living, growing tissue that contains about 1.5 million fibers at birth and about 3.5 million fibers in the eighth decade of life. ${ }^{1}$ In the adult lens, the lens epithelial cells migrate toward the bow region of the lens, where they differentiate into lens fiber cells. Throughout the life span of the individual, this process of differentiation and growth of the lens occurs. During this normal aging process, there are increases in light scattering, coloration, and fluorescence.

When the structure of the lens is modified so that it scatters light in the forward direction and this degree of increased scattering interferes with normal vision, we define the region of increased light scatter as a cataract. Cataract is usually defined as an opacity in the lens. This condition can be treated surgically.

Several techniques are available to noninvasively observe the human lens. Specular microscopy has been developed to image the human and animal lens and to observe the cells in the lens epithelium. ${ }^{2,3}$ In order to quantitate the manifestations of the aging lens, several noninvasive techniques have been developed: they include quasielastic light scattering, fluorescence and Raman spectroscopy, and Scheimpflug photography. ${ }^{4-6}$

The Scheimpflug principle, when adapted to a Scheimpflug camera yields photographs of the full thickness of the human lens. ${ }^{7}$ Line scans across these images can be used to quantitate the light scattering in the ocular lens. ${ }^{7}$ The Scheimpflug principle is illustrated in Figure 1. The development of a rotating Scheimpflug based on a Topcon SL-45 camera was first developed to obtain planar images of light scatter from various meridians of the lens. ${ }^{8}$ The technique of reconstructing three-dimensional figures from a set of Scheimpflug images acquired along various meridians was first demonstrated by Emori et al. ${ }^{9}$ These authors built up a threedimensional reconstruction of the lens from a series of coronal sections. Sakamoto and Sasaki ${ }^{10}$ have 


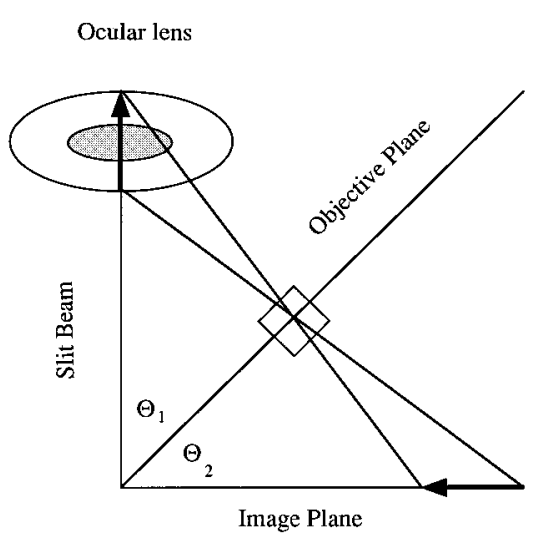

Fig. 1 The principle of the Scheimpflug camera. The plane containing the slit beam and the plane containing the image plane meet at one point, with the corresponding angles $\theta_{1}$ and $\theta_{2}$ identical. The corresponding angles are the angles between the objective plane, which contains the camera objective, and the planes containing the slit beam and the image. A Scheimpflug image of the lens is formed in the image plane.

used the same technique to image the threedimensional lens.

The paper describes the use of a rapid technique to visualize the full volume of the lens as a series of orthogonal slices through the full volume of the reconstructed lens. The use of the Dicer ${ }^{\circledR}$ software on a Macintosh computer permits rapid rendering of the slices $(10 \mathrm{~s} /$ slice). This work differs from previous three-dimensional studies in the following aspects: (1) the use of a new interpolation procedure to convert the meridian sections into a reconstructed full volume of the lens; (2) the use of readily available, inexpensive, commercial software to visualize the lens; and (3) use of pseudocolor to aid in the visualization of lens opacities. The images have been converted to gray scale for printing in this journal.

\section{METHODS}

\subsection{IMAGE ACQUISITION}

The Scheimpflug images were provided by Dr. K. Sasaki, Department of Ophthalmology, Kanazawa Medical University, Kanazawa, Japan. A set of 60 Scheimpflug images were acquired with the Anterior Eye Segment Analysis System (Nidek, EAS1000). A typical Scheimpflug image of scattered light through the cornea and the ocular lens is shown in Figure 2. This new type of Scheimpflug camera is based on a CCD camera with a computer analysis system. The Scheimpflug camera was rotated about the optic axis of the patient's eye in 3 deg increments to obtain 60 images, which sampled the full volume of the in vivo human ocular lens. In the clockwise rotation, image number 1 was obtained parallel to the superior-inferior axis of the globe; image number 31 was in a plane orthogonal to the plane used to acquire image number 1; and

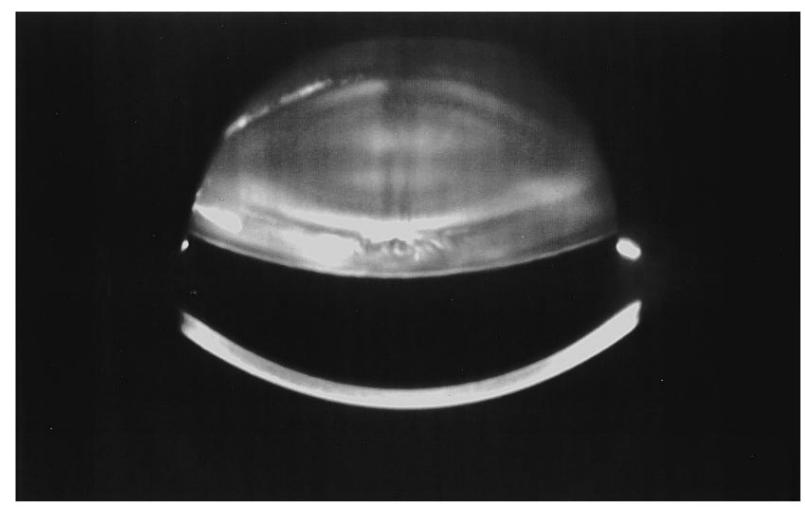

Fig. 2 A Scheimpflug image from the set of 60 images used for the three-dimensional reconstructions. The image shows the variation in light scatter within the plane containing the cornea and the ocular lens. The two-dimensional image shows the anterior lens surface, areas of opacity within the lens, and the posterior lens surface. Between the cornea and the lens are two bright reflections from the iris.

image number 60 was parallel to the inferiorsuperior plane of the eye. Twenty minutes were required to acquire the images. Each digital image was 640 by 400 pixels. The digital images had a dynamic range of 8 bits or 256 gray levels.

\subsection{IMAGE PROCESSING}

The acquired Scheimpflug images were not in registration due to eye movements during the image acquisition process so the images were realigned interactively. One image was chosen as the "fiducial" (vertical meridian at $0 \mathrm{deg}$ ) and two landmarks adjacent to the two iris reflections were identified on that section. A corresponding pair of landmarks was picked on an adjacent section. The alignment technique consisted of an $x-y$ shift (to superpose the midpoints of the two line segments), a rotation (to superpose the line segments), and a scaling (to superpose the pairs of points themselves). Application of these transformations to each pixel in the target image resulted in registered images. This set of operations was applied successively to the 60 Scheimpflug images in the data set.

\subsection{IMAGE RESAMPLING}

Image resampling refers to the procedure for converting the aligned set of 60 rotated Scheimpflug images into a new set of 332 images by a bilinear interpolation method. ${ }^{11}$ The new set of images was orthogonal to the optic axis, and consisted of slices from the anterior poles to the posterior poles of the ocular lens.

\subsection{OPTICAL TOMOGRAPHY}

The three-dimensional ocular lens was visualized using the interactive software program Dicer ${ }^{\circledR}$ from Spyglass, Inc. (Champaign, Illinois). This program was originally developed at the National Center for Supercomputing Applications of the University of 
Illinois at Urbana-Champaign. ${ }^{12}$ The program permits the display of three dimensional volume data as a set of orthogonal slices, oblique slices, blocks, or cutouts. It has been used to visualize such diverse applications as astrophysics, and ocean modeling, as well as biomedical applications. The advantages of the software include the ability to perform the visualization on a Macintosh II computer and its rapid speed of visualization. On a Macintosh Quadra model 950, the full data set of the in vivo human lens is $70 \mathrm{MB}$ and $10 \mathrm{~s}$ is required to draw the full color image of any plane through the volume.

The Dicer ${ }^{\circledR}$ software package, which is available for the PC, Macintosh, or Unix workstations, requires that the data set be in hierarchical data format (HDF). ${ }^{13}$ A data conversion utility supplied as part of DICER ${ }^{\circledR}$ rapidly converted the slices in the tagged image file format (TIFF) format into the HDF format, which is read by the DICER ${ }^{\circledR}$ software. HDF is an extensible, binary, public domain file format specification for storing data and images. It was also developed by the National Center for Supercomputing Applications and is used for all National Aeronautics and Space Administration space imaging applications. It supports both gray scale and pseudocolor tables to help visualize the volume. HDF files can store floating point data, scaling information, color images, text annotation, and other items. HDF was developed as a solution to the problem of sharing data among different computer systems. The stack of two-dimensional images of the human lens was in TIFF. Animations of the volume can be saved as a sequence of HDF, PICT, or TIFF files. Since the program is interactive, the user has control over the creation and manipulation of slices, cubes, cutouts, image size and orientation, colors, shading, and transparency.

Optical tomography was performed in the following manner. The stack of 368 TIFF images was first converted into an HDF file using the Spyglass Data Utility. The resulting file was $70 \mathrm{MB}$. A full block of the data was created. Since the individual Scheimpflug images of in vivo human lenses are situated along the $z$ axis, it is useful to visualize slices through the lens that are orthogonal to the original Scheimpflug images. The sequential movement of this cutting plane from the anterior of the lens to the posterior capsule permits visualization of the opacities and light scatter in the volume of the lens. The resulting visualization of light scatter in the human lens could be viewed from any orientation. In addition to the view from the anterior viewpoint (looking from the pupil to the posterior lens surface), it was of interest to show views with the viewpoint at the posterior side of the lens. In these views the observer is positioned behind the posterior pole of the lens. Finally, some isometric views of the lens are shown. Video animations were also made showing moving cutting planes from the anterior to the posterior pole of the lens, as well as cutting planes moving in orthogonal directions. These views could not be visualized with standard Scheimpflug images and represent new viewpoints of the distribution of light scatter in the human lens.

\section{RESULTS}

The technique described in this paper can project many orthogonal or oblique slices through the volume of a three-dimensional data set containing the cornea and the ocular lens. These visualizations in three dimensions are best appreciated in live animations in which the cutting planes move from one side of the volume to the other. In order to illustrate this in still photographs, several views from the animations were selected. Two orthogonal projection planes containing two slices from the threedimensional volume are shown in isometric views. The intensity of the scattered light is coded in a gray scale from 0 to 255 intensity levels. It was found that subtle variations in intensity of scattered light are best appreciated when the volume is pseudocolored. Each of the individual Scheimpflug images is a slice through the cornea, the ocular lens from anterior pole to posterior pole. Light scatter from a portion of the iris is also contained in the single Scheimpflug images. When the volume is reconstructed, the full cornea, a circular ring from the iris, and the three-dimensional ocular lens is contained in the block. In order to visualize this dense three-dimensional structure, slices were projected into the volume. Four views of interest were selected from the multitude of possible views to illustrate the technique. In Figure 3 the cornea, iris, and regions of light scatter in the lens are shown. Figure 4 shows the ability to view the regions of lens opacity from both the anterior and posterior viewpoints. None of the images shown in Figures 3 and 4 could be seen in the standard Scheimpflug image of the lens.

\section{DiscUSSION}

Why is three-dimensional visualization of ocular tissues a useful technique? While the printed page may show either stereo pairs, or red-green stereo anaglyphs, the true power of three-dimensional visualization is realized in interactive visualization on a computer. This can be documented in computer animations or movies of rotating three-dimensional objects. Three-dimensional visualizations of the rabbit cornea, ${ }^{14-16}$ rabbit lens, ${ }^{17,18}$ and the human in vivo fundus and optic nerve ${ }^{19}$ have been developed from confocal optical slices of ocular tissue. These three-dimensional visualizations permit the observer to interactively view the ocular tissue from any arbitrary viewpoint. This permits observer viewpoints that are not possible from the original set of acquired slices, and results in improved understanding of three-dimensional tissues. 


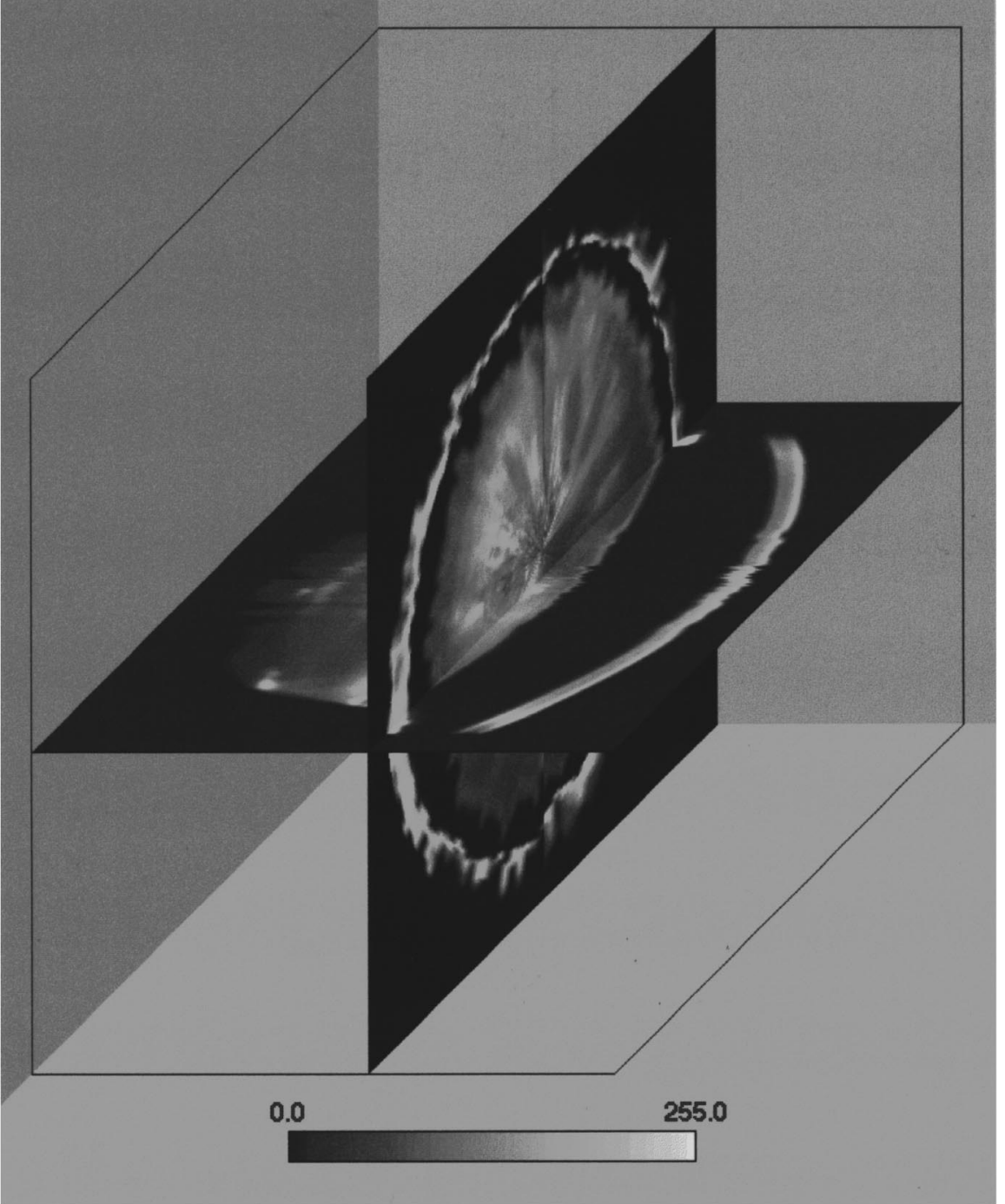

(a)

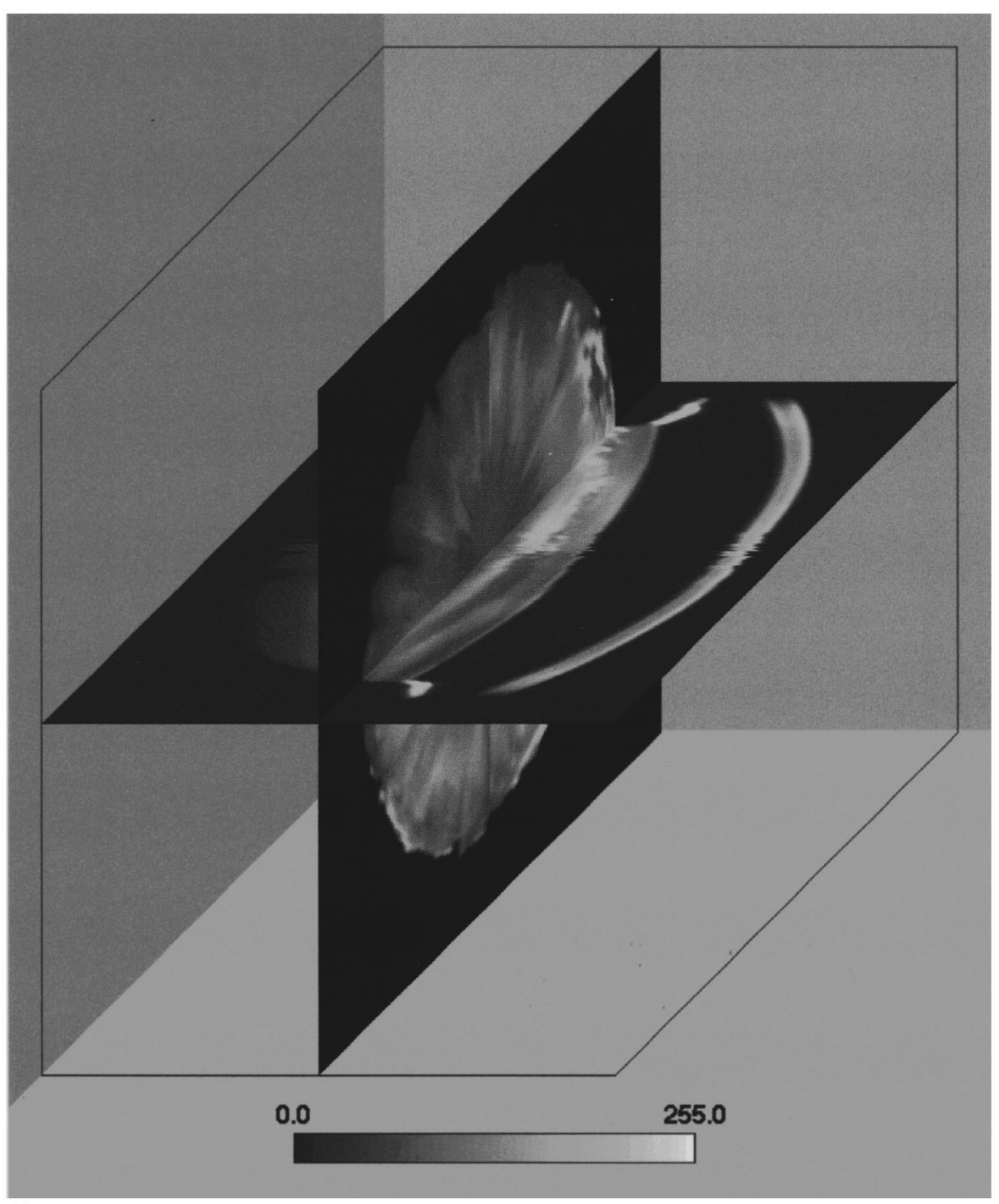

(b)

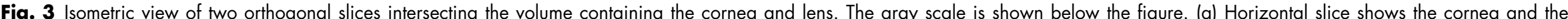

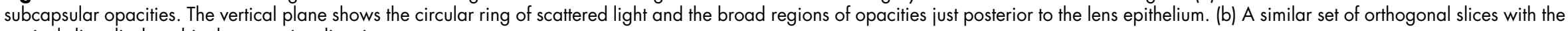
vertical slice displaced in the posterior direction. 


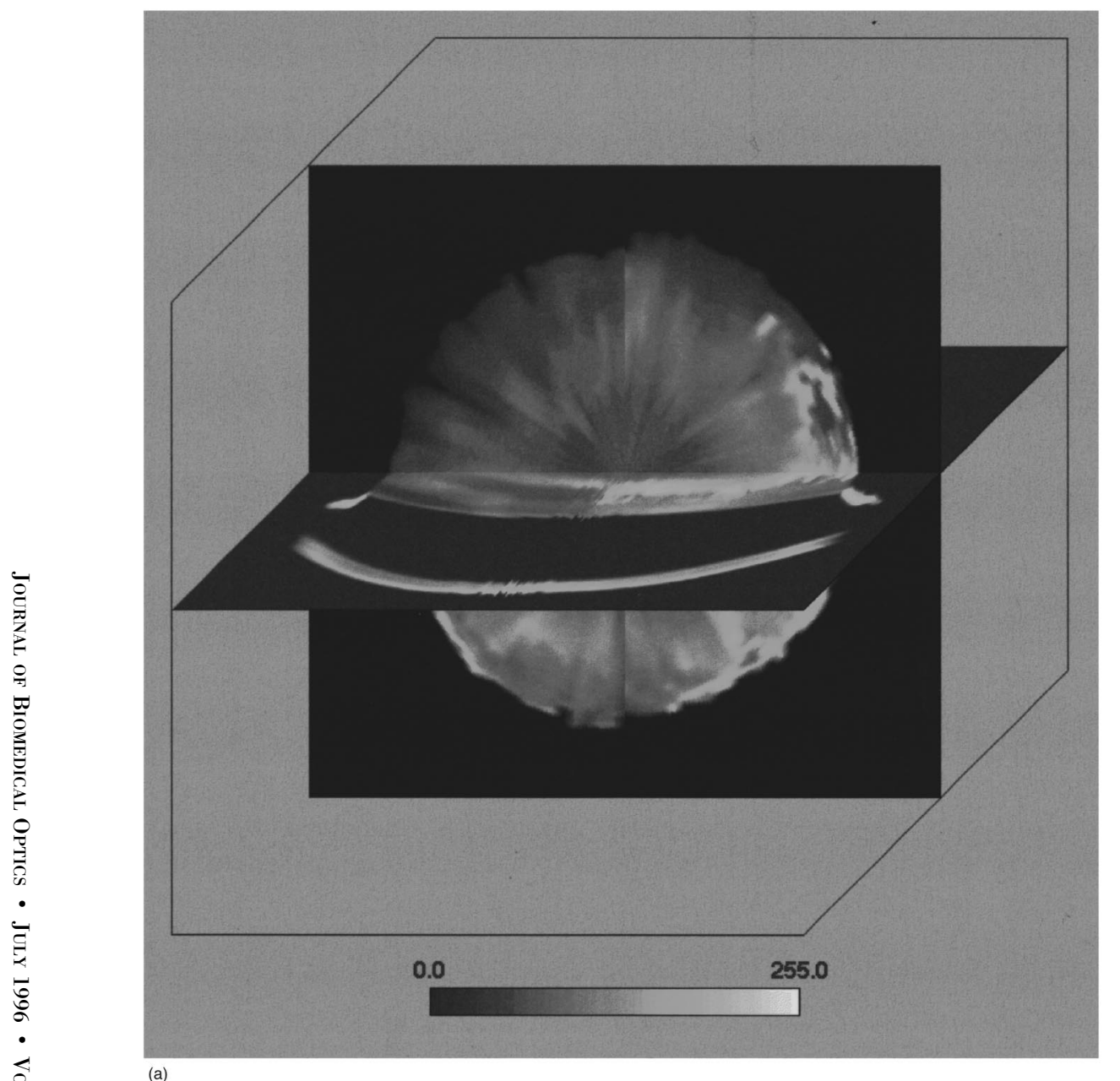

(a)

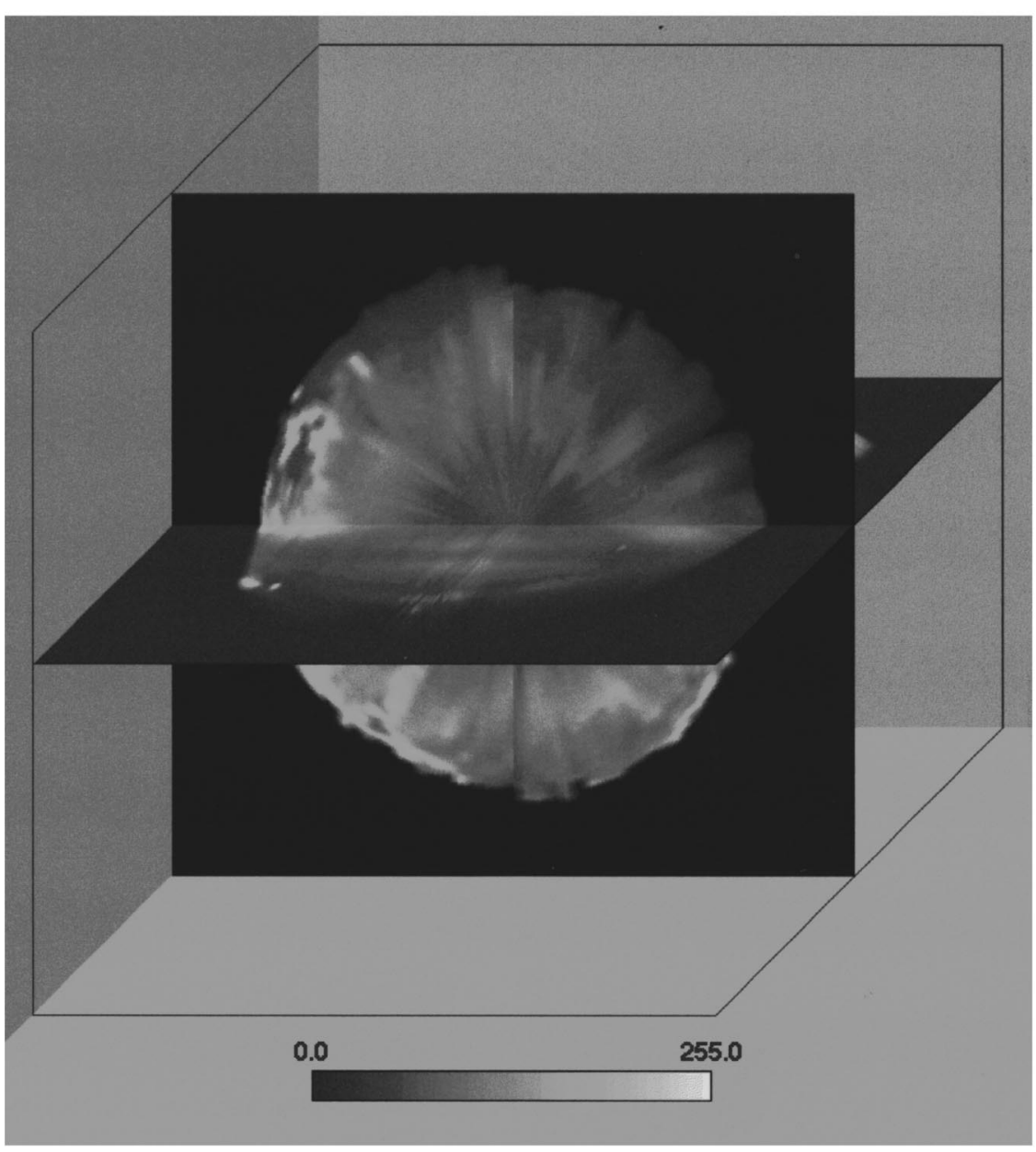

(b) 
What are the advantages of using threedimensional visualization techniques in evaluating cataract development with the Scheimpflug camera? Scheimpflug imaging of the lens has evolved from a single image, to several images taken in any meridian, to the full three-dimensional visualization of the ocular lens. While a single-slice image can indicate the existence of a cataract, it cannot provide information about its size, shape, and degree of opacification. The combination of a mechanically rotating Scheimpflug camera and imageprocessing techniques permits the visualization of the full volume of the in vivo human ocular lens. In principle, the volumes of each region of opacity and its shape could be determined from the analysis of the full volume of the lens.

This work differs from previous ${ }^{9,10}$ threedimensional studies in the following aspects: (1) use of a new interpolation procedure to convert the meridian sections into a reconstructed full volume of the lens; (2) use of readily available, inexpensive, commercial software to visualize the lens; and (3) use of pseudocolor to aid in the visualization of lens opacities. The three-dimensional visualization technique presented in this paper provides the full three-dimensional volume reconstruction directly, and the reconstructed images show high contrast and more detail of the scattering centers than were obtained with the previous methods.

At present, there are several technical problems to solve before the technique can become widespread in the clinic. The most severe problem is that the present Scheimpflug camera must be mechanically rotated to obtain the various images on each meridian of interest. This is a rather slow process and not suitable for routine clinical diagnostics because the 20 min time requirement for 60 images is not acceptable for the patient. The second problem is that there is optical distortion in the Scheimpflug camera used in this application. Finally, there is the question of calibration of the scattering and masking and shading errors. Since the light is incident on the lens from the anterior side, the presence of scattering regions will reduce both the incident light and the scattered light from more posterior regions.

The problem of mechanical rotation of the Scheimpflug camera can be solved by the use of a rapidly rotating optical system to acquire images in the various meridians. The inherent distortions in the Scheimpflug camera's optical system could be measured and then corrected. Finally, the masking and shading errors could be calibrated and corrected. These three solutions could transform the present mechanically rotating Scheimpflug camera into a clinical instrument for measuring light scatter in the in vivo human lens.

I suggest several new improvements to the current Scheimpflug camera. The use of an intensified CCD camera with $1024 \times 1024$ pixels, and 12 bits of dynamic range would improve image resolution and contrast. The improved dynamic range would result in improved quantitation of the opacities in the human lens. An intensified camera would improve the capability to detect small opacities. A true color CCD camera would permit the threedimensional visualization of human cataracts in true color, which is another parameter for the grading and characterization of cataracts.

The development of an instrument with the capability to visualize the light scatter from the full volume of the in vivo human lens would be a major advance in the diagnostics of cataract development. The increased acquisition speed, sensitivity, and dynamic range of the proposed system may result in understanding the development of lens opacities.

\section{Acknowledgment}

This work was supported by National Institutes of Health Grant EY-06958 (BRM).

\section{REFERENCES}

1. N. S. Jaffe and J. Horwitz, Lens and Cataract, Vol. 3, Textbook of Ophthalmology, S. M. Podos and M. Yanoff, Eds., Gower Medical Publishing, New York (1992).

2. A. J. Bron, "Specular microscopy of human and animal lenses in vivo and vitro," Ophthalmology 2, 229-232 (1985).

3. N. A. P. Brown and A. J. Bron, "An estimate of the human lens epithelial cell size in vivo, " Exp. Eye Res. 44, 899-906 (1987).

4. S-E. Bursell and N-T. Yu, "Fluorescence and Raman spectroscopy of the crystalline lens," in Noninvasive Diagnostic Techniques in Ophthalmology, B. R. Masters, Ed., pp. 319-341, Springer-Verlag, New York (1990).

5. S-E. Bursell, P. C., Magnante and L. T. Chylack, Jr., "In vivo uses of quasi-elastic light scattering spectroscopy as a molecular probe in the anterior segment of the eye," Noninvasive Diagnostic Techniques in Ophthalmology, B. R. Masters, Ed., pp. 342-365, Springer-Verlag, New York (1990).

6. T. Scheimpflug, "Der Photoperspektrograph und seine Anwendung," Photogr. Korr. 43, 516-531 (1906).

7. O. Hockwin, K. Sasaki, and S. Lerman, "Evaluating cataract development with the Scheimpflug camera," in Noninvasive Diagnostic Techniques in Ophthalmology, B. R. Masters, Ed., pp. 282-318, Springer-Verlag, New York (1990).

8. V. Dragomirescu, O. Hockwin, H. R. Koch, and K . Sasaki, "Development of a new equipment for rotating slit image photography according to Scheimpflug's principle," Interdis. Top. Gerontol. 13, 1-13 (1978).

9. Y. Emori, Y. Yasuda, H. Fukuda, and S. Sasaki, "Application of image processing technique for analyzing the optical system of the eye," in Advances in Diagnostic Visual Optics, A. Fiorentini, D. L. Guyton, and I. M. Siegel, Eds., pp. 55-60, Springer-Verlag, Berlin (1987).

10. Y. Sakamoto and K. Sasaki, "Computerized tomographic images and three-dimensional expression of crystalline lens findings from multiple slices of Scheimpflug slit images," Ophthalmic Res. 27 (Suppl. 1), 94-99 (1995).

11. B. R. Masters and S. L. Senft, "Transformation of a set of slices rotated on an axis to a set of z-slices," Comp. Med. Imag. Graphics (submitted).

12. P. J. Moran, "Nicer-slicer-dicer: an Interactive volume visualization tool," Proc. SPIE 2184, 214-222 (1994).

13. B. Fortner, The Data Handbook, A Guide to Understanding the Organization and Visualization of Technical Data, Spyglass, Inc., Champaign, IL (1992).

14. B. R. Masters and S. W. Paddock, "Three-dimensional reconstruction of the rabbit cornea by confocal scanning opti- 
cal microscopy and volume rendering," Appl. Opt. 29 (26), 3816-3822 (1990).

15. B. R. Masters and M. F. Farmer, "Three-dimensional confocal microscopy and visualization of the in situ cornea," Computerized Med. Imag. Graphics, 17 (3), 211-219 (1993).

16. B. R. Masters, "Confocal ocular microscopy: a new paradigm for ocular visualization," in Visualization in Biomedical Microscopies, A. Kriete, Ed., pp. 184-203, VCH, Weinheim, Germany (1992).

17. B. R. Masters, "Two-and three-dimensional confocal micros- copy of the eye," Multidimensional Microscopy, P. C. Cheng, T. H. Lin, W. L. Wu, and J. L. Wu, Eds., pp. 322-338, Springer-Verlag, New York (1993).

18. B. R. Masters, "Two- and three-dimensional visualization of the living cornea and ocular lens," Mach. Vis. Appl. 4, 227232 (1991).

19. F. W. Fitzke and B. R. Masters, "Three-dimensional visualization of confocal sections of in vivo human fundus and optic nerve," Curr. Eye Res. 12, 1015-1018 (1993). 